\title{
Efecto de la alimentación suplementaria con Plantago lanceolata sobre la oviposición de parásitos gastrointestinales en terneros
}

\author{
Effect of the supplementary feeding with Plantago lanceolata on the egg output \\ of gastrointestinal nematodes in calves \\ G Sievers $^{1 *}$, S Nannig ${ }^{2}$ \\ ${ }^{1}$ Instituto de Patología Animal, Universidad Austral de Chile, Valdivia, Chile. \\ ${ }^{2}$ Casilla: 103, Frutillar, Chile.
}

\begin{abstract}
SUMMARY
The purpose of this study was to evaluate whether Plantago lanceolata (narrow leaf plantain), as a supplemental forage, reduces the egg output of gastrointestinal nematodes in calves. The trial was conducted on a farm in the $10^{\text {th }}$ Region, Chile. Two groups of 15 calves weighing $171.4 \pm 19.8 \mathrm{~kg}$ grazed 17 hours a day on a pasture composed mainly of gramineous and with a high infestation of trichostrongylid nematode larvae. During the remaining 7 hours, the "control" group grazed in a pasture of similar botanic composition but with a low parasite burden; the "plantago" group grazed in a pasture specially sown with P. lanceolata with also a low parasite burden. This regimen was maintained for 60 days. Each week the animals were weighed and faecal samples were gathered to determine the nematode egg count (epg) and larvae generic differentiation. Grass samples were taken every two weeks from all pastures to establish their level of parasite burden. Precipitation was measured daily. The parasite burden of grass in the contaminated pasture was high during the whole period. Three weeks after the initiation of the trial, an increase in the average of eggs per gram of faeces (epg) was observed. A significant difference $(\mathrm{P}<0.05)$ in the averages of egg counts of Ostertagia and Trichostrongylus were observed from the 4th to the 7th week of the supplementation period, being lower in the Plantago supplemented group. All animals gained weight during the first 3 weeks, then the $P$. lanceolata supplemented group increased its weight by and average of $8.05 \mathrm{~kg}$ per animal more than the control group, however this difference was not significant $(\mathrm{P}>0.05)$. It is concluded that the inclusion of $P$. lanceolata as a supplemental forage significantly reduces the egg output of gastrointestinal parasites.
\end{abstract}

Palabras clave: terneros, Plantago, trichostrongilidos, hpg.

Key words: calves, Plantago, Trichostrongylidae, epg.

\section{INTRODUCCION}

Los nemátodos de la familia Trichostrongylidae provocan pérdidas económicas por atrasar el crecimiento, su futuro productivo y a veces causar la muerte de los bovinos. En el sur de Chile, con un clima mediterráneo templado, las especies parasitarias más frecuentes que afectan los terneros son: Ostertagia ostertagi, Cooperia oncophora, Cooperia spp., Trichostrongylus axei y Nematodirus helvetianus (Valenzuela 1988).

En el sur de Chile, los casos de parasitosis clínica se presentan en los terneros a fines de abril o inicios de mayo, coincidiendo con el inicio del período de lluvias otoñoinvernales (Sievers 1982); esto se debe a que la traslación de las larvas de las heces al pasto se ve favorecida por la lluvia (Gronvold y Hogh-Schmidt 1989, Sievers y col 1998), conllevando a una ingestión masiva de ellas por parte de los terneros. El otro momento en que se producen parasitosis clínicas es a fines de invierno, en agosto-septiembre, en bovinos que están cumpliendo el año de edad, coincidiendo con el aumento de la temperatura

Aceptado: 23.03.2006.

gsievers@uach.cl ambiental que favorece la maduración masiva de las larvas que permanecieron hipobióticas durante parte del otoño e invierno (Sievers 1982, Winkler 1999).

La resistencia antihelmíntica en el bovino, también descrita en Chile (Sievers y Fuentealba 2003), ha incentivado la búsqueda de nuevas alternativas de control de las parasitosis, y una de ellas es hacer pastorear a los bovinos sobre pasturas con especies vegetales que tengan un efecto antihelmíntico (Coop y Holmes 1996, Robertson y col 1995, Min y Hart 2003).

En Nueva Zelandia se ha evaluado la hierba Plantago lanceolata ("llantén de hoja angosta o siete venas") como una especie forrajera con potencial antihelmíntico, por ello se seleccionó una variedad con crecimiento erecto, mayor tamaño de hoja y frondosidad. P. lanceolata es una planta perenne, con crecimiento invernal, de hojas anchas acanaladas dispuestas en roseta, muy palatable y nutritiva para ovinos y bovinos. Posee niveles altos de minerales, destacando el calcio, cobre y cobalto. Alcanza una producción anual de materia seca (MS) similar a la de la ballica perenne, y se adapta muy bien a diferentes tipos de suelos, a condiciones de baja fertilidad y a sequías (Moorhead 2001). Esta planta contiene componentes biológicamente activos con propiedades antihelmínticas, antibacterianas, antiinflamatorias y 
antitumorígenas (Deacker y col 1994). Rumball y col (1997) describen extractos de $P$. lanceolata una actividad inhibitoria in vitro de la motilidad de larvas infectantes de T. colubriformis de ovinos. Sin embargo, aún no se tienen antecedentes sobre el efecto directo de $P$. lanceolata sobre los parásitos gastrointestinales en terneros a pastoreo (Stewart y col 1999).

A base de una importación de semilla seleccionada de $P$. lanceolata desde Nueva Zelandia, se programó el presente ensayo para evaluar si existe disminución de la oviposición de los parásitos en terneros.

\section{MATERIAL Y METODOS}

Ubicación del estudio: el ensayo se realizó en un predio situado de la X Región, Chile (41 ㅇ7'S; 7304'O), desde el 14 de abril al 14 de julio del 2003. En un sector de potreros de las vacas de lechería se prepararon previamente tres potreros de la siguiente forma:

1) Potrero "Llantén": en marzo del 2002, se sembraron 2,5 hectáreas con $7 \mathrm{~kg}$ por hectárea de semillas de la variedad comercial de $P$. lanceolata seleccionada. El potrero se fertilizó con nitrógeno, fósforo y potasio y se controlaron las malezas con herbicidas. Dicha superficie se contaminó con una baja carga parasitaria, haciendo pastorear 56 novillos infectados naturalmente con nemátodos durante tres días en febrero del 2003.

2) Potrero "Alta carga parasitaria": en 5 hectáreas de pradera natural mejorada se manejaron varias veces terneros positivos a nemátodos gastrointestinales, según la disponibilidad de forraje existente durante la primavera 2002 y verano 2003. De esa forma, dicha superficie se contaminó con una cantidad alta de huevos de trichostrongilidos. A inicios de abril de 2003, dicho potrero se subdividió en dos áreas de 2,5 hectáreas, que se denominaron A y B.

3) Potrero "Baja carga parasitaria": en 5 hectáreas de pradera natural mejorada pastorearon esporádicamente vacas de lechería en un régimen de rotación de potreros del predio. Como las vacas eliminan pocos huevos de parásitos (Sievers 1982), dicha superficie quedó con una "baja carga parasitaria". A inicios de abril de 2003, dicho potrero se subdividió en dos áreas (C y D) de 2,5 hectáreas.

Animales y grupos: para la realización de la prueba se utilizaron 30 terneros Overo Colorado, criados en forma artificial y estabulados durante 5 meses, se trasladaron una semana a un potrero con una empastada recién instaurada y exclusiva. El 14 de abril de 2003 se asignaron al azar dos grupos de 15 terneros de 171,4 $\pm 19,8$ kg; estos grupos se denominaron grupo "Plantago" y grupo "Testigo", los que pastorearon los potreros preparados mediante un cerco eléctrico siguiendo el siguiente esquema:

a) Período de infección parasitaria y suplementación de 60 días con $P$. lanceolata (24 de abril al 22 de junio de 2003): el Grupo Plantago se manejó 17 horas al día (de las 18 hrs de un día a las 11 hrs del día siguiente) sobre el área A del potrero "Alta carga parasitaria", y durante las 7 horas restantes (de las 11 a las 18 hrs) sobre el potrero "Llantén"; el Grupo Testigo se manejó respectivamente sobre el área B del potrero "Alta carga parasitaria" y luego en el área C del potrero "Baja carga parasitaria" en los mismos horarios y por igual período de tiempo. De esa forma se produjo una infección parasitaria diaria de ambos grupos comparables y que aseguró que la única diferencia entre los grupos de terneros fuera la alimentación suplementaria con $P$. lanceolata. La ración base para el Grupo Plantago fue: $3 \mathrm{~kg}$ de materia seca por ternero al día del área A y $3 \mathrm{~kg}$ de materia seca por ternero al día como ración suplementaria del potrero "Llantén". La ración base para el Grupo Testigo fue: $3 \mathrm{~kg}$ de materia seca por ternero al día del área B y 3 $\mathrm{kg}$ de materia seca por ternero al día y de pradera normal del área $\mathrm{C}$ del potrero "Baja carga parasitaria". La capacidad de consumo de pradera de los terneros se estimó en $6 \mathrm{~kg}$ de MS/día según la Tabla AFRC 1990, citada por Anrique y col (1995). La disponibilidad de MS/ha de cada potrero se evaluó cada 15 días cortando el pasto de 4 cuadrantes de $0,25 \mathrm{~m}^{2}$ y secándolo en un microondas.

b) Período final: durante 21 días (23 de junio al 14 de julio del 2003), ambos grupos se llevaron al área D del potrero "Baja carga parasitaria" donde el pastoreo se suplementó con heno a discreción.

Variables cuantificadas:

1) Composición botánica: una semana antes del inicio del ensayo se cortó el pasto de cuatro cuadrantes (de $0,25 \mathrm{~m}^{2}$ cada uno) en los potreros "Llantén", "Alta carga parasitaria" y "Baja carga parasitaria", para determinar la composición botánica general que se realizó en el Laboratorio de Nutrición Animal de la Universidad Austral de Chile. No se determinó la composición botánica específica de las praderas.

2) Carga parasitaria del pasto: cada dos semanas se tomaron 4 pequeñas muestras de pasto (de lo que cabe entre el pulgar y el índice), cada 10 pasos en las dos diagonales de cada potrero, formando dos muestras acumulativas según el procedimiento descrito por Kloosterman (1971), que luego se procesaron en el Laboratorio de Parasitología Veterinaria de la Universidad Austral de Chile, para determinar la cantidad de larvas infectantes por kilogramo de materia seca de pasto ( $\mathrm{L} / \mathrm{kg} \mathrm{ms}$ ), según la técnica descrita por Sievers (1973).

3) Oviposición de los parásitos: la cantidad promedio de huevos por gramo de heces (hpg) de los terneros del Grupo Plantago y del Grupo Testigo se determinó cada semana al realizar el análisis coproscópico individual a cada animal, según la técnica descrita 
por Schmidt (1971). Las muestras fecales se procesaron en el Laboratorio de Parasitología Veterinaria de la Universidad Austral de Chile.

4) Géneros de parásitos: con las muestras fecales de los dos grupos de terneros se realizaron cada semana dos cultivos acumulativos mediante la técnica de Roberts y O'Sullivan (1950) y las larvas infectantes se diferenciaron mediante la clave de Bürger y Stoye (1968).

5) Peso de los terneros: se determinó individualmente cada semana mediante una balanza electrónica.

6) Pluviosidad diaria en $\mathrm{mm}^{3}$ : se midió con un pluviómetro artesanal utilizado en el predio.

Análisis estadístico: Los pesos y la oviposición de los parásitos (hpg) se analizaron con el programa Statgraphics Plus, con un nivel de significancia $(\mathrm{P}<0,05)$. La interpretación de los resultados se basó en el Test de Cochran, el análisis de varianza (ANOVA) y el Test Multiple Range.

\section{RESULTADOS}

La composición botánica de los potreros "Alta y Baja carga parasitaria" fue similar, compuesta por gramíneas $(61 \%)$, leguminosas $(2,3 \%)$ y material muerto $(35,7 \%)$. En el potrero "Llantén" se encontró $P$. lanceolata $(67,3 \%)$, leguminosas $(7,5 \%)$ y material muerto $(23,3 \%)$.

El número de larvas infectantes por $\mathrm{kg}$ de materia seca de pasto ( $\mathrm{L} / \mathrm{kg} \mathrm{ms}$ ) en el potrero "Alta carga parasitaria”, en las áreas A y B, fluctuó entre 7.000 y
$4.000 \mathrm{~L} / \mathrm{kg}$ ms. En los potreros "Llantén” y "Baja carga parasitaria", el número de larvas en el pasto no superó las $280 \mathrm{~L} / \mathrm{kg} \mathrm{ms} \mathrm{(figura} \mathrm{1).}$

Durante el período de infección parasitaria y suplementación con $P$. lanceolata se determinó la oviposición de los siguientes géneros parasitarios (figura 2). Desde el 14 de abril al 12 de mayo de 2003, el único género que se diagnosticó fue Nematodirus, y su oviposición fue decreciente y similar en ambos grupos de terneros. A partir del 19 de mayo se inició la oviposición de Ostertagia y Trichostrongylus en ambos grupos de terneros siguiendo una tendencia creciente hasta el 23 de junio de 2003; del 26 de mayo al 23 de junio de 2003 la oviposición de ambos géneros fue superior en el grupo Testigo $(\mathrm{P}<0,05)$. La mayor reducción de oviposición la registró el género Ostertagia. El género Cooperia se presentó en ambos grupos con recuentos promedios muy bajos e irregulares. En el período final de observación, que se inició el 24 de junio del 2003, fecha en que concluye la suplementación de 60 días con $P$. lanceolata, y al mantener ambos grupos sobre el área D del potrero "Baja carga parasitaria", se igualan las oviposiciones de ambos géneros parasitarios $(\mathrm{P}>0,05)$.

El peso corporal de los dos grupos de terneros (figura 3) aumentó hasta el 26 de mayo de 2003. A partir del 2 de junio de 2003 se registró pérdida de peso en ambos grupos, siendo superior en el grupo Testigo. Al

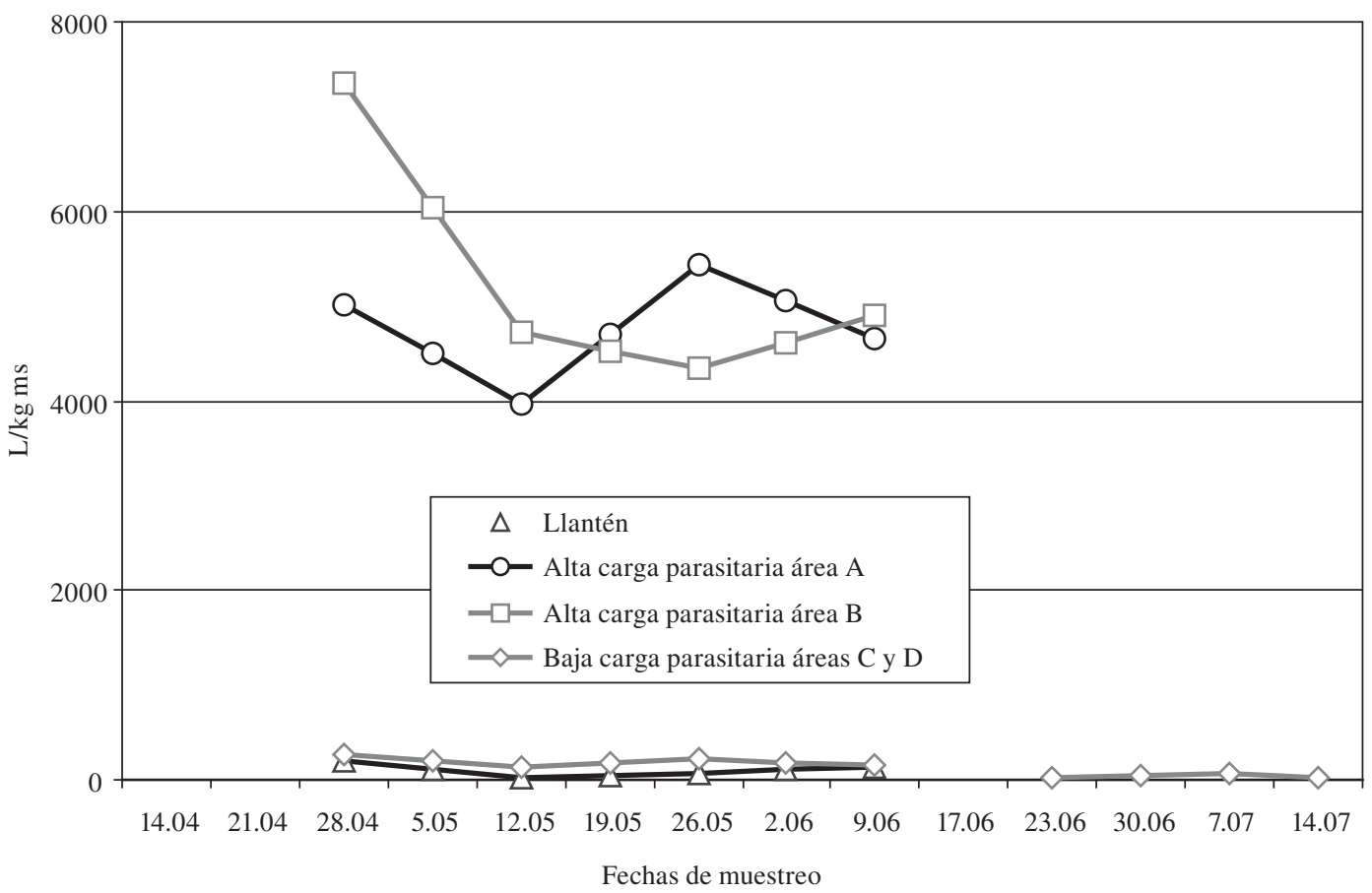

Figura 1. Larvas infectantes por kilogramo de materia seca de pasto ( $\mathrm{L} / \mathrm{kg} \mathrm{ms}$ ) en los potreros utilizados por los terneros durante un ensayo de suplementación con Plantago lanceolata.

Infective parasite larvae per kilogram of grass dry matter $(\mathrm{L} / \mathrm{kg} \mathrm{ms})$ in pastures used by the calves during a supplementation assay with Plantago lanceolata. 
ser manejados ambos grupos en el área D del potrero "Baja carga parasitaria" se recuperó la tendencia al aumento de peso de ambos grupos, pero el peso promedio de los terneros del grupo Plantago fue superior en 12,8 $\mathrm{kg}$. Sin embargo, no hubo diferencia significativa $(\mathrm{P}>0,05)$ entre ambos grupos.

Llovió sobre $50 \mathrm{~mm}$ en la semana previa al $20 \mathrm{de}$ abril de 2003; posteriormente hubo poca lluvia y entre las semanas del 9.06 al 22.06.03 se registró la mayor cantidad de agua caída de todo el período del ensayo.

\section{DISCUSION}

La composición botánica general de los potreros establecidos utilizados en el ensayo, con un predominio de gramíneas, corresponde a lo normalmente encontrado en los predios ganaderos del sur de Chile. El cálculo de la cantidad de forraje consumido diariamente por los animales se realizó a base de su densidad y disponibilidad (Anrique y col 1995), por ese motivo es posible asegurar que la única diferencia entre los dos grupos de terneros consistió en las 7 horas que un grupo pastoreó racionadamente el potrero en el cual predominaba P. lanceolata.

La preparación previa del potrero "Alta carga parasitaria" se realizó pragmáticamente haciendo pastorear varias veces grupos numerosos de bovinos jóvenes durante el período estival inmediatamente anterior y aseguró que los dos grupos de terneros del ensayo tuvieran una infección parasitaria básicamente similar. Al respecto debe aclararse que los valores arrojados por la técnica que determina la carga de larvas por kg de pasto seco de un potrero ( $\mathrm{L} / \mathrm{kg} \mathrm{ms}$ ) sólo son indicativos de una cantidad alta o baja y que las cifras no pueden analizarse como verdades absolutas. Son la resultante de aproximadamente 200 a 240 pequeñas muestras de pasto que conforman dos muestras acumulativas que se promedian para minimizar lo más posible la enorme variación de hojas de pasto que hay en una pastura de 2,5 hectáreas.

En el presente ensayo se esperó el inicio de las lluvias otoñales, que se produjeron en la semana del 20 de abril de 2003 en que llovieron $53 \mathrm{~mm}$, para asegurar que el pasto del potrero "Alta carga parasitaria" tuviera larvas en una cantidad suficiente como para provocar una parasitosis que afectara a los terneros. Al respecto, por experiencia personal no publicada del primer autor, el pastoreo constante de una superficie que tenga una cantidad superior a $2000 \mathrm{~L} / \mathrm{kg} \mathrm{ms}$ provoca en terneros pérdida de peso y diarrea. Ello permite asegurar que los dos grupos de terneros estuvieron sometidos a una infección alta al pastorear 17 horas diarias las áreas respectivas del potrero "Alta carga parasitaria".

Habiéndose cumplido el período prepatente de tres semanas (del 28 de abril al 19 de mayo del 2003), se produjo el inicio de la oviposición de Ostertagia y

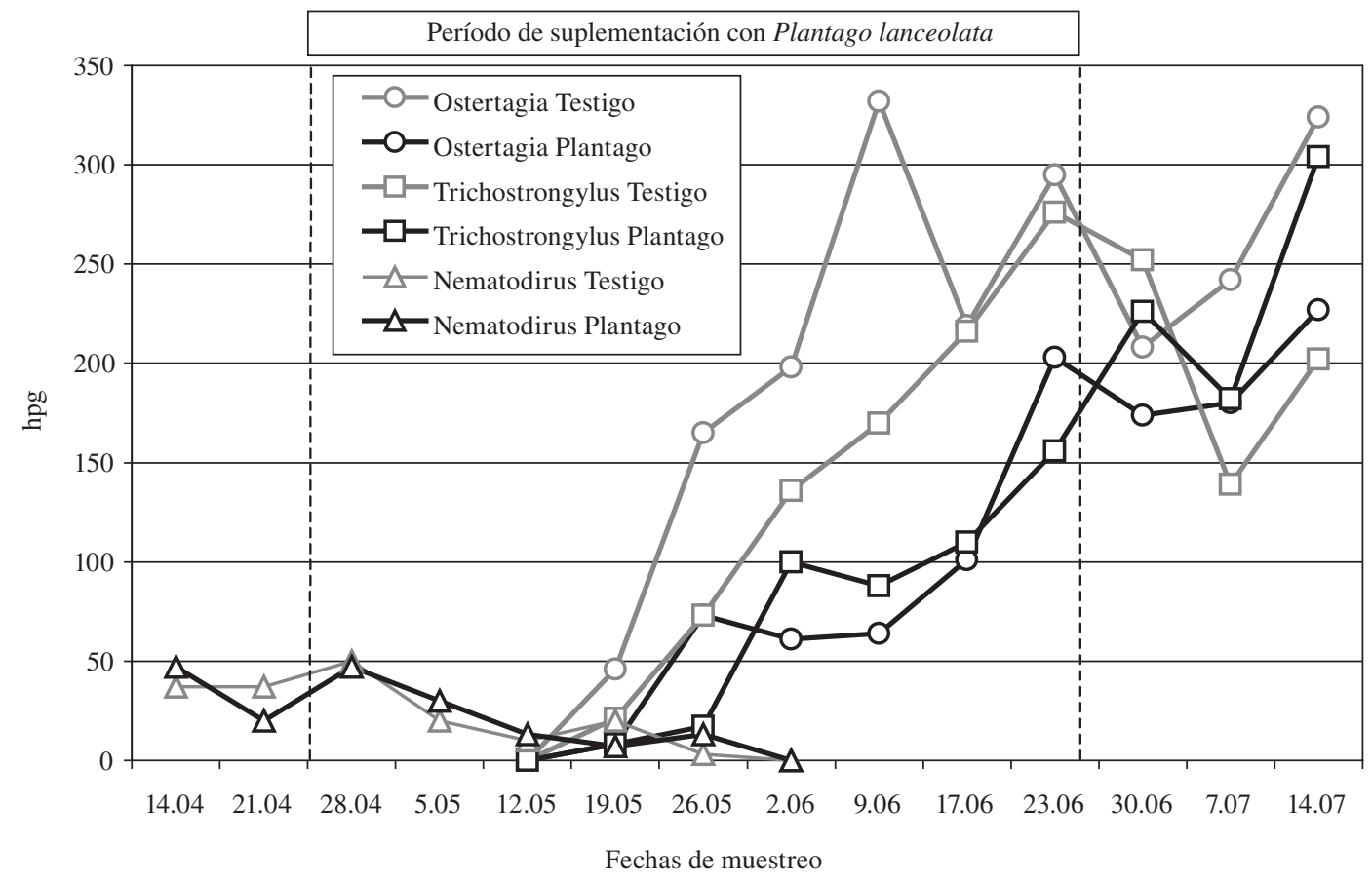

Figura 2. Recuentos promedio de huevos por gramo (hpg) de Ostertagia, Trichostrongylus y Nematodirus en heces de dos grupos de terneros, uno de ellos suplementado con Plantago lanceolata durante 60 días.

Mean egg-count per gram (epg) of Ostertagia, Trichostrongylus and Nematodirus in faeces of two groups of calves, one of them supplemented with Plantago lanceolata for 60 days. 


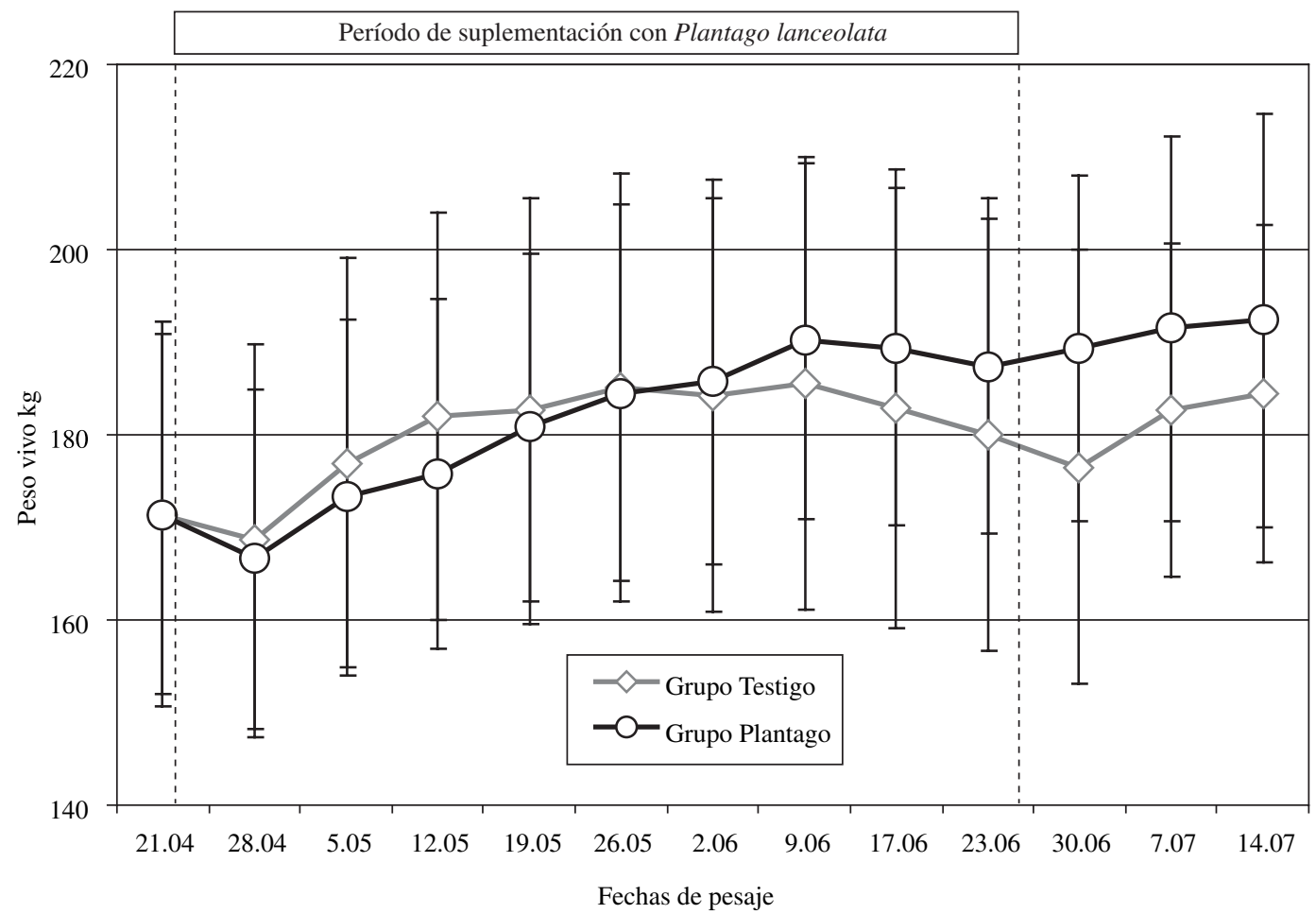

Figura 3. Variación del promedio y desviación estándar de peso corporal $(\mathrm{kg})$ de dos grupos de terneros, uno testigo y el otro suplementado con Plantago lanceolata durante 60 días.

Variation of mean body weight $(\mathrm{kg})$ and standard deviation in two calve groups, one control and the other supplemented with Plantago lanceolata for 60 days.

Trichostrongylus que, a grandes rasgos, aumentó hasta el 23 de junio (figura 2). Es necesario recordar que los recuentos de huevos expresados en hpg son sólo un reflejo de una población de parásitos desconocida y no son indicativos de un mayor o menor número de parásitos en el interior de un ternero o grupo de ellos; los hpg sólo indican una mayor o menor contaminación que un grupo de animales está provocando en un área determinada. Sin embargo, la tendencia a perder peso corporal que mostraron ambos grupos de terneros en las tres primeras semanas del mes de junio (figura 3 ), y que fue más pronunciada en el grupo Testigo, indican que todos los animales estaban siendo afectados por la presión de infección parasitaria a la que estaban sometidos al pastorear las áreas respectivas del potrero "Alta carga parasitaria". A ello debe agregarse la observación subjetiva, lamentablemente no cuantificada, de las heces líquidas y del aspecto deteriorado que mostraron los terneros del grupo Testigo, que permite a los autores indicar que esos animales estaban siendo más afectados por los parásitos que el grupo de terneros que estaba siendo suplementado con $P$. lanceolata.

Con respecto a la diferenciación de la oviposición de los parásitos (figura 2), la presencia inicial y desaparición del género Nematodirus, en ambos grupos de terneros, puede considerarse como una infección previa en el potrero destinado inicialmente a ellos; su evolución coincide con la tendencia normal para animales de esa edad y esa época del año (Winkler 1999). No se pudo visualizar una diferencia entre las oviposiciones de Nematodirus en los dos grupos de terneros. Llama la atención la bajísima presencia del género Cooperia, porque es un parásito que se encuentra regularmente en el ganado vacuno de la X Región de Chile (Valenzuela 1988). En cambio, la aparición y el constante aumento de huevos de Ostertagia y Trichostrongylus, a partir de la tercera semana del ensayo (19 de mayo del 2003), indican el éxito de la infección programada de ambos grupos de terneros. Sin embargo, los recuentos fueron siempre inferiores $(\mathrm{P}<0,05)$ en el grupo Plantago hasta el término del período de suplementación (23 de junio del 2003). Ello permite concluir que esta especie vegetal es responsable de la reducción de la oviposición de los parásitos, no concordando con el trabajo que describe que corderos que pastorearon una pradera de $P$. lanceolata presentaron recuentos superiores a otros grupos de corderos (Robertson y col 1995); pero cabe mencionar que en dicho trabajo no se mencionan ni la contaminación previa de las superficies de pastoreo ni la carga parasitaria de los mismos durante el ensayo.

En las tres semanas finales, posteriores al período de 60 días de suplementación, el grupo de terneros Testigo 
presentó una disminución de los recuentos promedio de huevos (figura 2), lo cual se puede atribuir a la baja carga de dicho potrero, así como también a la suplementación adicional con heno. A pesar de ello, la mayoría de los animales del grupo Testigo siguió presentando heces blandas e incluso un ternero tuvo diarrea profusa y fue necesario retirarlo del ensayo y tratar con un antinematódico. Al mismo tiempo todos los terneros del grupo suplementado con $P$. lanceolata presentaron heces de una consistencia semisólida normal. Esto sugiere que $P$. lanceolata, fuera de la capacidad de inhibir oviposición, mejora la condición general de los animales. La acción de la hierba sobre los parásitos albergados en el tracto gastrointestinal debe comprobarse en un ensayo clínico.

En relación al peso corporal promedio de los terneros (figura 3), ambos grupos aumentaron su peso hasta el día 26 de mayo de 2003. Que inicialmente los pesos promedios fueran algo inferiores en el grupo Plantago pudo deberse al cambio brusco de alimentación, ya que no hubo un período de adaptación previo. Luego esto se invirtió hasta el final del ensayo, siendo $12,8 \mathrm{~kg}$ superior la ganancia promedio de peso del grupo Plantago; sin embargo, no fue estadísticamente significativa $(\mathrm{P}>0,05)$ por la gran dispersión de los pesos individuales. Esta diferencia en el grupo Plantago se podría atribuir a un efecto de $P$. lanceolata sobre la población de los parásitos que lograron asentarse en el tracto gastrointestinal de los terneros.

Se concluye que la suplementación con $P$. lanceolata reduce la oviposición de los parásitos gastrointestinales.

\section{AGRADECIMIENTOS}

Muy en especial agradecemos al Sr. Rolf Nannig que permitió y organizó este trabajo en su predio.

\section{RESUMEN}

El propósito fue determinar si Plantago lanceolata ("Llantén de hoja angosta") ingerida como forraje suplementario reduce la oviposición de los nemátodos gastrointestinales en terneros. El ensayo se realizó en un predio de la X Región, Chile. Dos grupos de 15 terneros de $171,4 \pm 19,8 \mathrm{~kg}$, pastorearon 17 horas diarias un potrero contaminado con una alta carga de larvas de nemátodos trichostrongilidos. Las 7 horas restantes, el grupo de terneros "Testigo" pastoreó un potrero de igual composición botánica, pero con una baja carga parasitaria, y el grupo de terneros "Plantago" pastoreó un potrero sembrado con $P$. lanceolata, también con una baja carga parasitaria. Este manejo se mantuvo durante 60 días. Cada semana se pesaron todos los animales y se les tomaron muestras fecales para realizar recuento de huevos y diferenciación genérica de larvas. Cada dos semanas se estableció la carga parasitaria del pasto de los potreros utilizados. La pluviosidad se registró diariamente. La carga parasitaria del pasto del potrero contaminado fue alta durante todo el ensayo. A las tres semanas de iniciado el período de suplementación se inició en ambos grupos un aumento de los recuentos (hpg) promedio de Ostertagia y Trichostrongylus que se diferenciaron significativamente $(\mathrm{P}<0,05)$ desde la cuarta a la séptima semana, siendo inferiores en el grupo suplementado con $P$. lanceolata. Durante las primeras tres semanas todos los animales aumentaron de peso, luego el grupo de terneros "Plantago" tuvo un incremento de peso promedio superior en
$12,8 \mathrm{~kg}$ al grupo "Testigo", sin embargo, dicha diferencia no fue significativa $(\mathrm{P}>0,05)$. Se concluye que la suplementación con Plantago lanceolata redujo significativamente la oviposición de los parásitos gastrointestinales.

\section{REFERENCIAS}

Anrique R, X Valderrama, R Fuchslocher. 1995. Composición de alimentos para el ganado en la zona sur. Universidad Austral de Chile. Facultad de Ciencias Agrarias. Instituto de Producción Animal. Fundación Fondo de Investigación Agropecuaria, FIA, Ministerio de Agricultura, Pp 1-56.

Bürger HJ, M Stoye. 1968. Parasitologische Diagnostik (Teil II). Therapogen Praxidienst 3, 1-24.

Coop RL, PH Holmes. 1996. Nutrition and parasite interaction. Int $J$ Parasitol 26, 951-962.

Deacker JM, MJ Young, TJ Fraser, JS Rowarth. 1994. Carcass, liver and kidney characteristics of lambs grazing plantain (Plantago lanceolata), chicory (Cichorium intybus), white clover or perennial ryegrass. N Z Soc Anim Prod 54, 197-200.

Gronvold J, K Hogh-Schmidt. 1989. Factors influencing rain splash dispersal of infective larvae of Ostertagia ostertagi (Trichostrongylidae) from cow pats to the surroundings. Vet Parasitol 31, 57-70.

Kloosterman A. 1971. Observations on the epidemiology of trichostrongylosis of calves. H. Veenman and Zonen. N. V. Wageningen.

Min BR, SP Hart. 2003. Tannins for suppression of internal parasites. J Anim Sci 81, 102-109.

Moorhead A. 2001. Plantain-The Pasture Herb. PGG. Seeds Ceres Extension, Pp 1-9.

Roberts F, PJ O'Sullivan. 1950. Methods for eggs counts and larval cultives for strongylus infesting the gastrointestinal tract of cattle. Austr J Agr Res 1, 99-102.

Robertson HA, JH Niezen, GC Waghorn, WAG Charleston, M Jinlong. 1995. The effect of six herbages on liveweight gain, wool growth an faecal egg count of parasitised ewe lambs. Proc N Z Soc Anim Prod 55, 199-201.

Rumball W, RG Keogh, GE Lane, JE Miller, RB Claydon. 1997. Grasslands Lancelot plantain (Plantago lanceolata). N Z J Agr Res 40, 373-377.

Schmidt U. 1971. Parasitologische Kotuntersuchung durch ein neues Verdünnungsverfahren. Tierärztl Umsch 26, 229-230.

Sievers G. 1973. Methode zur Gewinnung von III. Strongylidenlarven aus dem Weidegras. Tesis de doctorado. Tierärztliche Hochschule Hannover. República Federal de Alemania.

Sievers G. 1982. Epizootiología y control de los parásitos de los rumiantes. VIII Jornadas Médico Veterinarias. Universidad Austral de Chile. Valdivia, Pp 93-112.

Sievers G, I Quintana, F Cortese, S Ernst. 1998. Variación anual de la ubicación de las larvas infectantes de tricostrongílidos del bovino sobre el pasto de un potrero en Valdivia, Chile. Arch Med Vet 30, 47-54.

Sievers G, C Fuentealba. 2003. Comparación de la efectividad antihelmíntica de seis productos comerciales que contienen lactonas macrocíclicas frente a nemátodos gastrointestinales del bovino. Arch Med Vet 35, 81-88.

Stewart A, M Urquhart, A Moorhead. 1999. Biologically active compounds present in plantain (Plantago lanceolata). Ceres Research Farm. P. O. Box 3100. Christchurch.

Valenzuela G. 1988. VII Día de campo ovino. Facultad de Ciencias Veterinarias. Universidad Austral de Chile. Valdivia, Pp 20-21.

Winkler M. 1999. Estudio sobre la eliminación de ooquistes, huevos y larvas de parásitos gastrointestinales y pulmonares en bovinos de carne en un predio de un valle cordillerano de la X Región de Chile. Tesis, M.V., Universidad Austral de Chile, Facultad de Medicina Veterinaria, Valdivia, Chile. 